\title{
Communication Strategies in Mohs Micrographic Surgery: A Survey of Methods, Time Savings, and Perceived Patient Satisfaction
}

Nyousha Yousefi, MD; Justin M. McLawhorn, MD; Andrew Quinn, MD; Alexander Stubblefield, MS; Sixia Chen, PhD; Thomas Stasko, MD; Lindsey Collins, MD

\section{PRACTICE POINTS}

- There are limited studies evaluating the efficacy of different communication methods in Mohs micrographic surgery (MMS) clinics.

- This study suggests that incorporation of a lightbased system into an MMS clinic improves workplace efficiency.
Mohs micrographic surgery (MMS) entails multiple time-consuming surgical and histological examinations for each patient. Efficient communication is key in improving clinic flow, and we surveyed members of the American College of Mohs Surgery to evaluate the efficacy of different techniques utilized by Mohs surgeons across the nation.

Cutis. 2021;107:249-252.

M ohs micrographic surgery (MMS) entails multiple time-consuming surgical and histological examinations for each patient. As surgical stages are performed and histological sections are processed, an efficient communication method among providers, medical assistants, histotechnologists, and patients is necessary to avoid delays. To address these and other communication issues, providers have focused on ways to increase clinic efficiency and improve patient-reported outcomes by utilizing new or repurposed communication technologies in their Mohs practice.

Prior reports have highlighted the utility of handsfree headsets that allow real-time communication among staff members as a means of increasing clinic efficiency and decreasing patient wait times. ${ }^{1-4}$ These systems may mediate a more rapid turnover between stages by mitigating the need for surgeons and support staff to assemble within a designated workspace. . $^{1,3,4}$ However, there is no single or standardized communication method that best suits all surgical suites and MMS practices. Our study aimed to identify the current communication strategies employed by Mohs surgeons and thereby ascertain which method(s) portend(s) the highest benefit in average daily time savings and provider-perceived patient satisfaction.

\section{Materials and Methods}

Survey Instrument-A new 10-question electronic survey was published on the SurveyMonkey website, and a link to the survey was provided in a quarterly email that originated from the American College of Mohs Surgery and was distributed to all 1735 active members. Responses were obtained from January 2019 to February 2019.

Dr. Yousefi is from the University of Michigan Medical School, College of Medicine, Ann Arbor. Drs. McLawhorn, Quinn, Chen, Stasko, and Collins as well as Mr. Stubblefield are from the University of Oklahoma Health Sciences Center, Oklahoma City. Drs. McLawhorn, Quinn, Stasko, and Collins are from the College of Medicine, Department of Dermatology, and Dr. Chen is from the Department of Biostatistics and Epidemiology. The authors report no conflict of interest. 
Statistical Analysis-A statistical analysis was done to determine any significant associations among the providers' responses. $P<.05$ was used to determine statistical significance. A Cochran-Armitage test for trend was used to identify significant associations between the number of rooms and the communication systems that were used. Thus, 7 total tests -1 for each device (whiteboard, light system, flag system, wired intercom, wireless intercom, walkie-talkie, or headset)—were conducted. The Cochran-Armitage test also was used to determine whether the probability of using the device was affected by the number of stations/surgical rooms that were attended by the Mohs surgeons. To determine whether the communication devices used were associated with higher patient satisfaction, a $\chi^{2}$ test was conducted for each device (7 total tests), testing the categories of using that device (yes/no) and patient satisfaction (yes/no). A Fisher exact test of independence was used in any case where the proportion for the device and patient satisfaction was $25 \%$ or higher. To determine whether the communication method was associated with increased time savings, 7 total Cochran-Armitage tests were conducted, 1 for each device. A logistic regression model was used to determine whether there was a significant association between the number of stations and the likelihood of reporting patient satisfaction.

\section{Results}

Eighty-eight surgeons responded to the survey, with a response rate of $5 \%(88 / 1735)$. A total of 55 surgeons completed the survey in its entirety and were included in the data analysis. The most commonly used communication mediums were whiteboards (29/55 [53\%]), followed by a flag system (16/55 [29\%]) and a light system (13/55 [24\%]). Most Mohs surgeons (52/55 [95\%]) used the communication media to communicate with their staff only, and 76\% (42/55) of Mohs surgeons believed that their communication media contributed to higher patient satisfaction. Overall, 58\% (32/55) of Mohs surgeons stated that their communication media saved more than 15 minutes (on average) per day. The use of a whiteboard and/or flag system was reported as the least efficient method, with average daily time savings of 13 minutes. With the introduction of newer technology

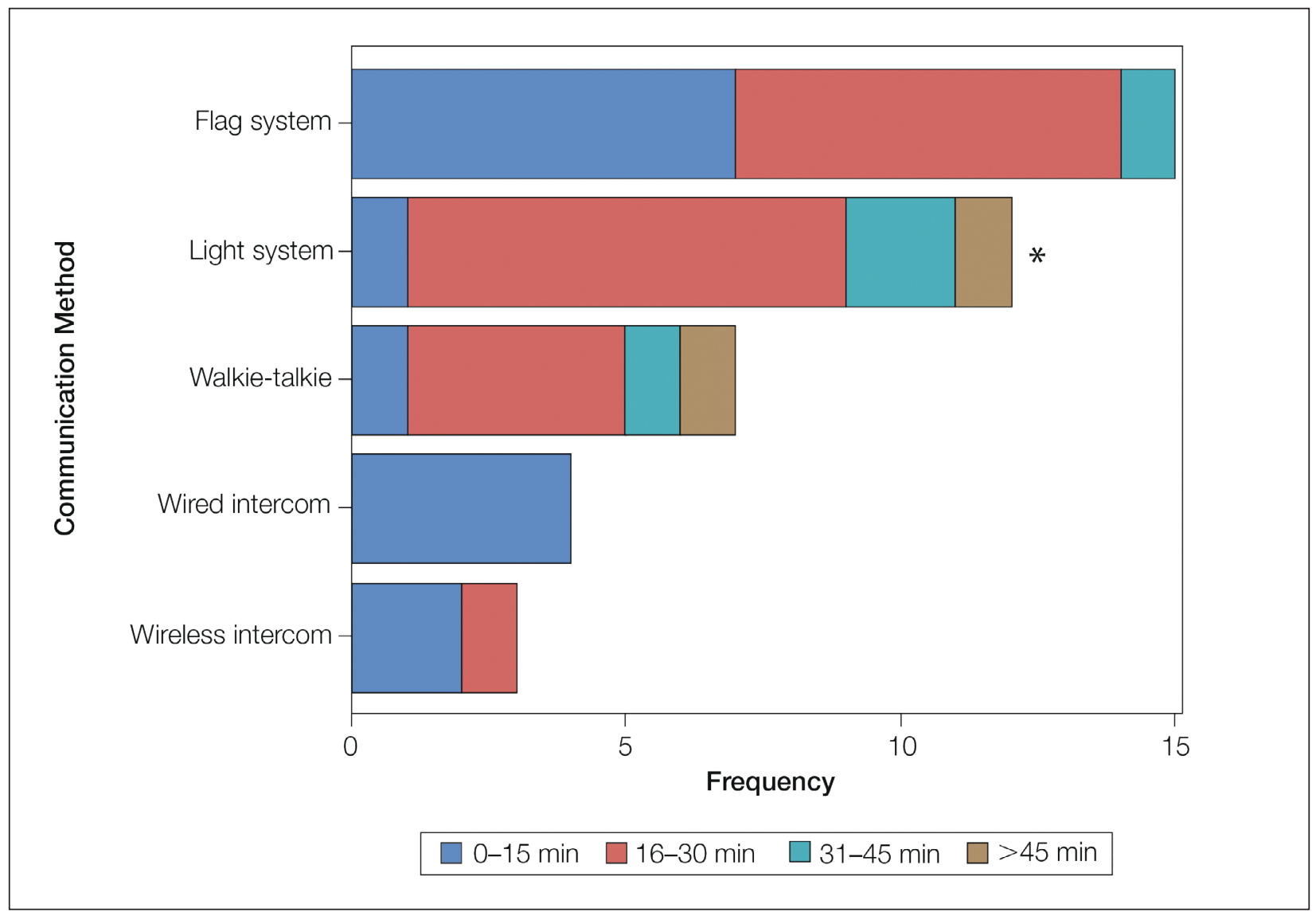

Provider-reported time savings of communication methods. This graph illustrates the communication media that were associated with an increase in time savings. Whiteboards and headsets were excluded because they did not increase time savings. The prevalence of each method (indicated by frequency) was further stratified by range of time savings, wherein the area of each stratification corresponded to the percentage of time savings indicated by the Mohs surgeons. Asterisk indicates $P=.0482$. 


\section{Communication Strategy Survey Results $(\mathrm{N}=55)$}

\section{Question}

Do you use any type of whiteboard, lighting system, flag system, radio, wired/wireless intercom, walkie-talkie, or headset for communication as part of your practice?

What is the medium that you use to communicate (check all that apply)?

How many stations/surgical rooms or devices are utilized?

With whom do you communicate using these communication media?

At any given point, approximately how many individuals have access to the communication medium at your facility?

How many patients do you see in a typical day while using this technology?

\section{Response}

Yes, 100\% (55/55); no, 0\%
Whiteboard, 53\% (29/55); flag system, 29\% (16/55);

light system, 24\% (13/55); wired/wireless intercom, 11\% (6/55); walkie-talkie, 13\% (7/55); headset, $11 \%(6 / 55)$; other, $13 \%(7 / 55)$

1-5 stations, 51\% (28/55); 6-10 stations, 35\% (19/55); $11-15$ stations, $13 \%$ (7/55); 16-20 stations, 0\%; $>20$ stations, $2 \%(1 / 55)$

Patients only, 0\%; staff only, 95\% (52/55); both, 5\% (3/55)
1-5 people, 27\% (15/55); 6-10 people, 55\% (30/55);

$11-15$ people, $9 \%(5 / 55) ; 16-20$ people, 5\% (3/55);

21-25 people, 0\%; 26-30 people, 0\%; >30 people, 4\% (2/55)

$1-5$ patients, $4 \%$ (2/55); 6-10 patients, 22\% (12/55);

11-15 patients, 15\% (8/55); 16-20 patients, 27\% (15/55);

21-25 patients, $11 \%(6 / 55) ; 26-30$ patients, $15 \%(8 / 55)$;

31-35 patients, 2\% (1/55); 36-40 patients, 0\%;

$>40$ patients, $6 \%(3 / 55)$

\section{In your assessment, do you believe that the} communication media employed at your facility contributes to higher patient satisfaction?

How much time per day do you estimate that you save by using your communication media?
Yes, $76 \%$ (42/55); no, 24\% (13/55)

$<0$ min, 4\% (2/55); 0-15 min, 38\% (21/55);

16-30 min, 38\% (21/55); 31-45 min, 12\% (7/55);

$>45 \mathrm{~min}, 8 \%(4 / 55)$

$\mathrm{PHI}$ is not transmitted, 56\% (31/55); encryption, 5\% (3/55); nothing, 10\% (5/55); not applicable, 29\% (16/55)
How do you safeguard patients' PHI while utilizing your communication system?

Abbreviation: $\mathrm{PHI}$, protected health information.

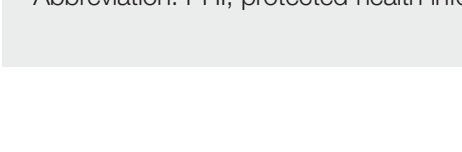

(wired or wireless intercoms, headsets, walkie-talkies, or internal messaging systems such as Skype) to the whiteboard and/or flag system, the time savings increased by 10 minutes per day. Nearly 25\% (14/55) of surgeons utilized more than 1 communication system.

As the number of stations in an MMS suite increased, the probability of using a whiteboard to track the progress of the cases decreased. There were no statistically significant associations identified between the number of stations and the use of other communication devices (ie, flag system, light system, wireless intercom, wired intercom, walkie-talkie, headset). The stratified percentages of the amount of time savings for each communication modality are presented in the Figure (whiteboards and headsets were excluded because they did not increase time savings). The use of a light system was the only communication modality found to be statistically associated with an increase in provider-reported time savings $(P=.0482$; Figure). In addition, our analysis did not show an improvement in provider-reported patient satisfaction with any of the current systems used in MMS clinics.

\section{Comment}

The process of transmitting information among the medical team during MMS is a complex interplay involving the relay of crucial information, with many opportunities for the introduction of distraction and error. Despite numerous improvements in the efficiency of the preparation of histological specimens and implementation of various time-saving and tissue-saving surgical interventions, relatively little attention has been given to address the sometimes chaotic and challenging process of organizing 
results from each stage of multiple patients in an MMS surgical suite. ${ }^{5}$

As demonstrated by our survey, incorporation of a light-based system into an MMS clinic may improve workplace efficiency by decreasing the redundant use of support staff and allowing Mohs surgeons to transition from one station to the next seamlessly. Light-based communication systems provide an immediate notification for support staff via color-coded and/or numerically coded indicators on input switches located outside and inside the examination/surgery rooms. The switch indicators can be depressed with minimal disruption from station to station, thereby foregoing the need to interrupt an ongoing excision or closure to convey the status of the case. These systems may then permit enhanced clinic and workflow efficiency, which may help to shorten patient wait times.

Study Limitation-Although all members of the American College of Mohs Surgery were invited to participate in this online survey, only a small number $(\mathrm{N}=55)$ completed it in its entirety. Moreover, sample sizes for some of the communication devices were small. As a result, many of the tests might be lacking sufficient power to detect possible relationships, which might be identified in future larger-scale studies.

\section{Conclusion}

Our study supports the use of light-based communication systems in MMS suites to improve efficiency in the clinic. Based on our analysis, light-based communication methods were significantly associated with improved time savings $(P=.0482)$. Our study did not show an improvement in provider-reported satisfaction with any of the current systems used in MMS clinics. We hope that this information will help guide providers in implementing new communication techniques to improve clinic efficiency.

Acknowledgments-The authors would like to thank Ms. Kathy Kyler (Oklahoma City, Oklahoma) for her assistance in preparing this manuscript. Support for Dr. Chen and Mr. Stubblefield was provided through National Institutes of Health, National Institute of General Medical Sciences [Grant 2U54GM104938-06, PI Judith James].

\section{REFERENCES}

1. Chen T, Vines L, Wanitphakdeedecha $R$, et al. Electronically linked: wireless, discrete, hands-free communication to improve surgical workflow in Mohs and dermasurgery clinic. Dermatol Surg. 2009;35:248-252.

2. Lanto $A B$, Yano EM, Fink A, et al. Anatomy of an outpatient visit. An evaluation of clinic efficiency in general and subspecialty clinics. Med Group Manage J. 1995;42:18-25.

3. Kantor J. Application of Google Glass to Mohs micrographic surgery: a pilot study in 120 patients. Dermatol Surg. 2015;41:288-289.

4. Spurk PA, Mohr ML, Seroka AM, et al. The impact of a wireless telecommunication system on efficiency. J Nurs Admin. 1995;25:21-26.

5. Dietert JB, MacFarlane DF. A survey of Mohs tissue tracking practices. Dermatol Surg. 2019;45:514-518. 